\title{
Joint Statement by the Surgery Journal Editors Group
}

We, the editors of surgery journals, believe that conducting sex-inclusive biomedical and clinical research is imperative to improving health outcomes of men and women. Note that the word "sex" is being used rather than "gender". Sex is the genotype by which one is born, and gender is the phenotype. It is the chromosomal sex of the human, animal, tissue, or cell to which we are referring. Recent studies have shown that the majority of biomedical research in the field of surgery and related topics is conducted on male animals and male cells, even when studying diseases prevalent in women [1]. Human clinical research suffers from a lack of sex-based reporting and sexbased analysis of the results [2,3]. Given these findings, the National Institutes of Health has now asked that sex be considered as a biologic variable in all National Institutes of Health-funded research [4]. As such, we support uniform, defined reporting of the sex used for human, animal, tissue, and cell research in ALL manuscripts published in our journals. If only one sex is studied, authors must include a justification statement as to why a single-sex study was conducted. We also will require sex-based reporting and analysis of data for all human, animal, tissue, and cell research. As a group, we will require this among all our collective surgery journals.

\section{References}

1. Yoon DY, Mansukhani NA, Stubbs VC, Helenowski IB, Woodruff TK, Kibbe MR (2014) Sex bias exists in basic science and translational surgical research. Surgery 156(3):508-516

2. U.S. Government Accountability Office. National Institutes of Health: better oversight needed to help ensure continued progress including women in health research. https://www.gao.gov/pro ducts/GAO-16-13. Published: Oct 22, 2015. Publicly Released: Oct 23, 2015

3. Mansukhani NA, Yoon DY, Teter KA, Stubbs VC, Helenowski IB Woodruff TK, Kibbe MR (2016) Determining if sex bias exists in human surgical clinical research. JAMA Surg 151(11):1022-1030

4. National Institutes of Health. Consideration of sex as a biological variable in NIH-funded research. https:/grants.nih.gov/grants/ guide/notice-files/NOT-OD-15-102.html. Published June 9, 2015
The Surgery Journal Editors Group is comprised of editors from 74 international, surgery-related journals who meet once a year at the annual meeting of the American College of Surgeons and discuss concerns common among surgery journals. The World Journal of Surgery editorial leadership is a member of this group and subscribes to the tenets expressed in the Joint Statement. Our instructions to authors will reflect these requirements and our editorial decisions will include their consideration. 